IdeAs

Idées d'Amériques

16 | 2020

Les marges créatrices : intellectuel.le.s afrodescendant.e.s et indigènes auX Amériques, XIX-XXe siècle

\title{
Le Parti démocrate en 2020 : Joe Biden et l'hydre à quatre têtes
}

\section{Christophe Cloutier-Roy}

\section{OpenEdition}

Journals

Édition électronique

URL : http://journals.openedition.org/ideas/9643

DOI : $10.4000 /$ ideas.9643

ISSN : $1950-5701$

Éditeur

Institut des Amériques

\section{Référence électronique}

Christophe Cloutier-Roy, «Le Parti démocrate en 2020 : Joe Biden et l'hydre à quatre têtes », IdeAs [En ligne], 16 | 2020, mis en ligne le 01 octobre 2020, consulté le 18 octobre 2020. URL : http:// journals.openedition.org/ideas/9643; DOI : https://doi.org/10.4000/ideas.9643

Ce document a été généré automatiquement le 18 octobre 2020

\section{(c) (1) $(9)$}

IdeAs - Idées d'Amériques est mis à disposition selon les termes de la licence Creative Commons Attribution - Pas d'Utilisation Commerciale - Pas de Modification 4.0 International. 


\title{
Le Parti démocrate en 2020 : Joe Biden et l'hydre à quatre têtes
}

\author{
Christophe Cloutier-Roy
}

I am not a member of any organized party. I am a

Democrat.

Will Rogers

1 Les conventions nationales des partis démocrate et républicain qui se sont tenues au mois d'août ont exposé de profondes différences entre les deux grandes formations politiques américaines, et pas uniquement sur le plan idéologique. Du côté des républicains, on a vu un parti qui semble désormais entièrement entre les mains du président Donald Trump, comme en a fait foi l'absence de la plupart des figures de l'establishment républicain et des anciennes gloires de ce parti. Celles-ci ont cédé leur place aux partisans les plus convaincus du $45^{\mathrm{e}}$ président des États-Unis et aux membres de sa famille, qui ont pris la parole tour à tour pour s'adresser à un auditoire de plus en plus restreint. En effet, sous Trump, le Grand Old Party, ou GOP, ne cherche même pas à prétendre représenter l'ensemble de la nation. Son message s'adresse exclusivement à son électorat blanc, conservateur et religieux, concentré dans les petites villes, les banlieues résidentielles et les campagnes. Or, la fameuse «majorité silencieuse » qui a jadis fait les beaux jours du GOP sous Nixon, Reagan et les Bush l'est de moins en moins, majoritaire. Devant cet entêtement des républicains à ne pas chercher à recruter audelà de leur électorat naturel, le Parti démocrate semble être dans une position enviable puisque, dans un système marqué par un bipartisme rigide, il est le seul à pouvoir récolter les fruits des pratiques d'exclusion du GOP. Or, le parti de l'âne vit en quelque sorte une situation inversée par rapport à son vis-à-vis : il a certes le potentiel d'attirer une majorité des électeurs américains, mais il doit pour cela trouver une façon d'accommoder des dizaines de groupes de pression aux priorités bien distinctes, une tâche qui doit être répétée à chaque cycle électoral.

2 Cette situation n'est pas neuve en soi. Fondé à la fin des années 1820 sur les ruines de l'ancien Parti démocrate-républicain de Thomas Jefferson et James Madison, le Parti démocrate est le premier parti politique moderne de l'histoire des États-Unis et le plus 
vieux parti politique toujours en activité dans le monde. Créé à l'origine pour défendre les droits des agriculteurs et des petits commerçants contre les élites financières, le parti a progressivement élargi sa base, devenant notamment le parti des immigrants et du Sud au cours de la seconde moitié du xix ${ }^{\mathrm{e}}$ siècle, puis celui des Afro-Américains, des ouvriers et des habitants des grandes villes à partir des années 1930, à quoi s'ajoutent les Hispaniques au tournant du XxI $\mathrm{I}^{\mathrm{e}}$ siècle. Tous ces groupes (à l'exception des Sudistes) continuent de peupler la grande tente démocrate aux côtés des jeunes, des femmes, des minorités culturelles, religieuses et sexuelles, des écologistes, des professions intellectuelles et artistiques et des militants de gauche. Plus que jamais, le Parti démocrate est une formation aux identités multiples, dont l'équilibre est constamment à rétablir. L'humoriste Will Rogers (1879-1935) disait qu'en tant que démocrate, il n'était membre d'aucun parti organisé. Il disait également: «Les démocrates ne sont jamais d'accord sur quoi que ce soit, c'est pour cela qu'ils sont démocrates. S'ils étaient d'accord, ils seraient républicains ». Plus de 80 ans après sa mort, ces pointes humoristiques sont plus à-propos que jamais. Pour décrire le Parti démocrate actuel, on pourrait utiliser l'image d'une formidable hydre à trois têtes. Ces trois têtes, qui représentent les trois identités du parti, ont pour nom "groupes d'intérêts", «minorités » et "gauche» et rugissent indépendamment les unes des autres. C'est cette bête puissante et indocile que doivent harnacher le candidat Joe Biden et sa colistière Kamala Harris, défi d'autant plus grand qu'en 2020 une quatrième tête a fait son apparition, celle de la résistance au trumpisme.

\section{Le parti des groupes d'intérêt}

Dans son ouvrage The End of Liberalism paru en 1969, le politologue américain Theodore Lowi affirmait que le libéralisme (terme utilisé ici dans son acception américaine au sens de progressisme) promu par le Parti démocrate était voué à s'écraser sous le poids du clientélisme du parti, dont il dénonçait la propension à accommoder les demandes du moindre des groupes d'intérêts militant en son sein. C'est d'ailleurs à Lowi qu'on doit l'expression « libéralisme de groupes d'intérêts » faisant référence à un libéralisme axé sur la satisfaction des besoins de groupes d'intérêts particuliers. Quoi que l'on pense de la justesse de la critique de Lowi, il est naturel qu'un parti fondé par accrétion de divers groupes d'intérêts au cours de son histoire en soit venu à laisser une grande place à ces groupes qui rivalisent entre eux pour influencer le contenu du programme démocrate. Ces rivalités internes ont souvent par le passé pris la forme d'affrontements intra-partisans qui ont pu miner la capacité des démocrates à présenter un front uni. Dès la fin des années 1940, le parti a commencé à se déchirer sur la question raciale entre les Blancs du Sud partisans de la ségrégation et le mouvement naissant pour les droits civiques. Cette première fissure s'est doublée au cours des années 1960 d'une dissension croissante sur la question du Vietnam. Ce n'est qu'à la fin de la décennie que les groupes favorables aux droits civiques et opposés à la Guerre du Vietnam se sont imposés définitivement, au prix de la perte du Sud et du démantèlement de la coalition du New Deal qui avait assuré la domination nationale du Parti démocrate depuis le début des années 1930. Aujourd'hui, les groupes d'intérêts sont encore légion à l'intérieur du Parti démocrate et, si les tensions internes ne sont pas aussi vives qu'elles l'ont été au cours des années 1960, le fait que ces groupes puisse poursuivre des objectifs contradictoires représente un danger pour l'unité du parti. À titre d'exemple, l'importance des groupes écologistes à l'intérieur du Parti démocrate a forcé la main de 
Joe Biden pour annoncer un programme ambitieux sur le plan environnemental, mais les propositions qu'il met en avant doivent tenir compte des préoccupations des syndicats, qui craignent qu'une transition énergétique trop rapide se traduise par des pertes d'emplois pour leurs membres.

\section{Le parti des minorités}

Fondé comme le parti de "l'homme ordinaire " (common man), le Parti démocrate est naturellement devenu au fil du temps le parti de tous ceux qui se situent en marge des pouvoirs économiques. Les minorités ethniques, religieuses et sexuelles y occupent aujourd'hui une place importante. À ces groupes, on pourrait ajouter les femmes, qui n'ont pas atteint la pleine égalité économique et politique avec les hommes et qui votent majoritairement pour le Parti démocrate depuis les années $1980^{1}$. Les jeunes aussi, sous-représentés dans les cercles de décision, constituent une force vive de la base du parti, particulièrement depuis les années Obama. En faisant une place à ces différents groupes, le Parti démocrate s'est assuré du soutien d'un bassin potentiel d'électeurs pouvant lui conférer un avantage décisif à moyen terme dans une nation dont les tendances démographiques pointent vers un rajeunissement et une diversification de la population. Or, cet appui amène avec lui deux défis majeurs : d'une part, la nécessité de multiplier les efforts pour s'assurer que ces électeurs participent aux élections, effort d'autant plus vital que dans de nombreux États, les législatures contrôlées par les républicains ont multiplié des mesures (diminution du nombre de bureaux de vote, nécessité d'amener plusieurs pièces d'identité officielles pour voter) qui rendent difficile l'exercice du droit de vote, particulièrement pour les minorités. D'autre part, le Parti démocrate doit composer avec les attentes de ces groupes en termes de représentativité, un enjeu qui est pratiquement absent à l'intérieur du Parti républicain. Lors des primaires de 2020, après avoir fait grand cas de la présence parmi les candidats à l'investiture démocrate de six femmes, de quatre Afro-Américains, d'un Sino-Américain, d'un Hispanique, d'un homosexuel et d'une Samoane, la victoire d'un candidat septuagénaire blanc a entraîné une profonde insatisfaction chez plusieurs militants et a poussé Joe Biden à annoncer dès le mois de mars qu'il choisirait une femme comme colistière. Si nul ne remet aujourd'hui en question les compétences de sa colistière Kamala Harris (la sénatrice de la Californie d'ailleurs issue des communautés afro- et indo-américaine), un tel engagement ouvre la porte pour accuser les démocrates de pratiquer le tokénisme ${ }^{2}$, ce dont ne se sont pas privé les républicains.

\section{Le parti de la gauche}

5 À partir du tournant du $\mathrm{xx}^{\mathrm{e}}$ siècle, le Parti démocrate a entamé un virage vers la gauche qui se reflète toujours dans les orientations politiques de ce parti. Sous l'impulsion du populiste William Jennings Bryan (trois fois candidat malheureux à la présidence en 1896, 1904 et 1908), puis des présidents Woodrow Wilson (1913-1921), Franklin Delano Roosevelt (1933-1945) et Harry Truman (1945-1953), le Parti démocrate a progressivement intégré l'idée que les ressources de l'État pouvaient être mobilisées pour niveler les inégalités socioéconomiques. Sous Lyndon Johnson (1963-1969), le Parti démocrate a mis en place la Great Society, un imposant ensemble de mesures visant à lutter contre la pauvreté et mettre fin à la discrimination raciale. Cet imposant 
programme politique a alimenté une vive réaction au sein de la population qui conduira éventuellement à la "révolution conservatrice " de Ronald Reagan (1981-1989) et poussera les Démocrates à se recentrer politiquement. On assiste toutefois à partir du milieu des années 2000 au retour en force d'une gauche militante à l'intérieur du Parti démocrate. En fait foi le succès inattendu, lors des primaires démocrates de 2016, de la candidature du sénateur indépendant Bernie Sanders (Vermont), qui se décrit comme un démocrate socialiste. Le retour en grâce de la gauche s'accompagne de tensions croissantes entre certains militants et l'establishment, les premiers préconisant l'adoption de mesures ambitieuses pour garantir l'accès à la santé et à l'éducation, pour lutter contre les changements climatiques ou pour encadrer les relations entre les policiers et les communautés ethnoculturelles, tandis que les seconds prêchent la modération au nom de l'impératif de ne pas rebuter des électeurs indépendants modérés dont l'appui est capital lors des élections.

\section{Le parti de la résistance au trumpisme}

6 En 2020, une nouvelle tête a poussé sur l'hydre démocrate, celle de l'opposition à Donald Trump. De manière paradoxale, le Parti démocrate a bénéficié de la victoire surprise de Trump en 2016. Certes, le parti a perdu la Maison-Blanche, mais la vive réaction causée par l'arrivée au pouvoir d'une figure aussi polarisante que celle du $45^{\mathrm{e}}$ président des États-Unis a entraîné un regain de vie pour un Parti démocrate qui n’avait pas cessé de perdre du terrain lors des huit années de la présidence Obama. Sans Trump à la Maison-Blanche, on peut difficilement imaginer comment les démocrates auraient pu reprendre le contrôle de la Chambre des représentants au terme des élections de mi-mandat de 2018.

7 Aujourd'hui, l'impopularité chronique de Trump permet aux démocrates d'entretenir l'espoir réaliste de le priver d'un second mandat, et ce, en dépit de l'avantage historique dont bénéficie le président en vue de sa réélection. Les résultats des scrutins et les sondages récents montrent que le Parti démocrate progresse auprès de clientèles électorales traditionnelles du Parti républicain, dont les femmes blanches des banlieues résidentielles, déçues de la position du GOP sur la question des armes à feu, ou encore les personnes âgées de plus de 65 ans qui reprochent au président Trump sa gestion de la crise de la COVID-19. On a même vu d'anciens élus et cadres du Parti républicain tel que l'ancien gouverneur de l'Ohio John Kasich prendre publiquement position contre Trump et soutenir Joe Biden. Le Parti démocrate ne peut toutefois pas se construire uniquement en opposition à un seul homme. Ainsi, au-delà d'une aversion partagée envers le président, ses coups d'éclat et ses politiques, comment intégrer dans la grande famille démocrate des républicains et des indépendants anti-Trump dont l'appui au-delà du départ du président n'est nullement garanti ?

\section{Joe Biden, dompteur de l'hydre démocrate?}

Depuis le début du cycle électoral actuel, les faiblesses de la candidature de Joe Biden ont souvent été mises en avant par les commentateurs de la scène politique américaine, qui ne se privent pas de souligner l'âge avancé (77 ans) de l'ancien vice-président d'Obama, sa propension à faire des gaffes et son absence de charisme. Si ces critiques ne 
sont pas infondées, il est tout de même regrettable que ces observateurs ne prennent pas la peine de souligner une des grandes réussites de la campagne de Biden jusqu'à maintenant, soit sa capacité à unifier le Parti démocrate en dépit des tensions internes. Le candidat a mis en avant des engagements qui prennent en considération les attentes des principaux groupes d'intérêts de la coalition démocrate, il s'est assuré de tendre la main aux groupes minoritaires (particulièrement la communauté afro-américaine) et s'est avéré une figure rassurante pour les républicains et les indépendants anti-Trump prêts à appuyer les démocrates le temps d'une élection.

9 La plus grande faiblesse de Biden demeure sa capacité à rallier l'aile gauche du parti. Pour remédier à cette faiblesse, au terme des primaires, il a rapidement opéré un rapprochement avec Bernie Sanders et la sénatrice du Massachusetts Elizabeth Warren, deux candidats défaits lors des primaires et des personnalités en vue de la gauche démocrate. La campagne de Biden a travaillé de concert avec celle de Sanders pour mettre en place ce que plusieurs décrivent déjà comme le programme le plus progressiste de l'histoire moderne du Parti démocrate. Lors de la convention nationale du parti, les démocrates ont montré un visage uni derrière leur candidat, un contraste par rapport au rendez-vous de 2016 quand les partisans déçus de Bernie Sanders avaient fait connaître leur mécontentement. Cette unité est précaire, mais il est permis de croire que Joe Biden, aidé de sa colistière Kamala Harris, a réussi pour l'instant à dompter l'hydre quadricéphale démocrate.

\section{NOTES}

1. Il convient de souligner qu'on parle ici d'une majorité de l'ensemble des femmes, tout groupe ethnique confondu. En 2016, une majorité de femmes blanches ont voté pour le républicain Donald Trump.

2. De l'anglais tokenism, pratique consistant à inclure des groupes minoritaires de manière superficielle et symbolique.

\section{AUTEUR}

\section{CHRISTOPHE CLOUTIER-ROY}

Détenteur d'un doctorat en science politique de l'Université du Québec à Montréal, Christophe Cloutier-Roy est chercheur à l'Observatoire sur les États-Unis de la Chaire Raoul-Dandurand en études stratégiques et diplomatiques. Ses recherches portent principalement sur les institutions politiques américaines, le Parti démocrate, les élections aux États-Unis et la relation canado- 
américaine. Il est fréquemment invité à analyser l'actualité américaine dans les médias québécois. 